\title{
SEVQER: Automatic Semantic Visual Query Builder to Support Intelligent Image Search in Traffic Images
}

\author{
Hui-Hui Wang, Phei-Chin Lim, Yin-Chai Wang, \\ Soo-See Chai, Dayang Nurfatimah Awang Iskandar and Wee Bui Lin \\ Department of Computing and Software Engineering, \\ Faculty of Computer Science and Information Technology, \\ Universiti Malaysia Sarawak, Kota Samarahan, Sarawak, Malaysia
}

\author{
Article history \\ Received: 01-02-2018 \\ Revised: 27-02-2018 \\ Accepted: 31-07-2018 \\ Corresponding Author: \\ Hui-Hui Wang \\ Department of Computing and \\ Software Engineering, Faculty \\ of Computer Science and \\ Information Technology, \\ Universiti Malaysia Sarawak, \\ Kota Samarahan, Sarawak, \\ Malaysia \\ Email: hhwang@unimas.my
}

\begin{abstract}
Image search is a challenging process in the field of Content Based Image Retrieval (CBIR). Image search-by-example, search-bykeyword and search-by-sketch methods seldom provide user interface that allows user to accurately formulate their search intent easily. To overcome such issue, a novel image search interface-Semantic Visual Query Builder (SeVQer) is proposed as a non-verbal interface which allows user to drag and drop from the image data provided to formulate user query. The drag and drop mechanism minimizes the difficulty of verbalizing query image into keywords or sketching a correct drawing of the query image. SeVQer was implemented and compared with 3 image search methods (search-by-example, search-by-keyword and search-by-sketch) in terms of task completion time and user satisfaction using traffic images. SeVQer achieved statistically significant lower task completion time with an average of $28 \mathrm{sec}$, a promising $50 \%$ reduction than search-by-sketch (average of $56 \mathrm{sec}$ ). The significance of this work is two-fold: the SeVQer user interface allows user to easily formulate intent specific query, while the novel architecture and methodology reduces the semantic gap in general.
\end{abstract}

Keywords: Intention Gap, Semantic Visual Query, Image Search Interface, Semantic-Based Image Retrieval

\section{Introduction}

Image retrieval is a very active research area with two main challenges - semantic gap and intention gap. The semantic gap is an interpretation inconsistency between low level visual features and high-level semantics (Wang et al., 2006; Smeulders et al., 2000) while intention gap is the gap between the users' search intents and the corresponding search queries (Zha et al., 2010; Cox et al., 2000) as illustrated in Fig. 1. The intention gap is the subset of semantic gap and is associated to the design of user interface of an image search system that allows users to effectively express their search intent. However, researches done on user interface design and support for CBIR systems (Pečenovió et al., 2000; Santini and Jain, 2000; Nakazato et al, 2003) are scarce.

Different users perceived and understood an image differently (Yoon, 2011), making understanding the user context for image searching process an essential step in designing an image retrieval system (Choi, 2010). In attempts to minimize the semantic gap between the users and image retrieval systems (Gudivada and Raghavan, 1995), query mechanism plays a vital role. A search query is commonly used by a user to express his or her search intent and information needs to retrieve images from image database that fit his or her perception. The submitted user queries affected directly the image search results and accuracy (Croft and Thompson, 1987). One of the major challenges is the utilization of semantic meaning and representation in user search queries in order to accurately describe the intention of the user search queries as well as the search domain (Hu et al., 2009).

The query formulation problem is where user can imagine what they desire but is unable to properly formulate or translate their information needs 
effectively (Urban and Jose, 2006; Kelly and Fu, 2007; Belkin, 2000) in precise wording (Chang et al., 2009). Typically, users uses not more than three words (Jansen et al., 2000) in forming search queries mainly because of the difficulty in expressing their information needs and due to the user interfaces of traditional search engines encourage them to do so (Belkin et al., 2003). In addition, the variation of different query languages also causes a great extent of images with high similarity but inaccurate semantically (Ménard, 2011). Keywords queries are usually short and ambiguous and thus hard to reflect user search intent precisely (Gerrig and Zimbardo, 2001). As a result, it is critical to understand and study how users express their search needs, which may ultimately lead to providing the pathway to fully utilizing visual materials for retrieval (Choi and Rasmussen, 2003).

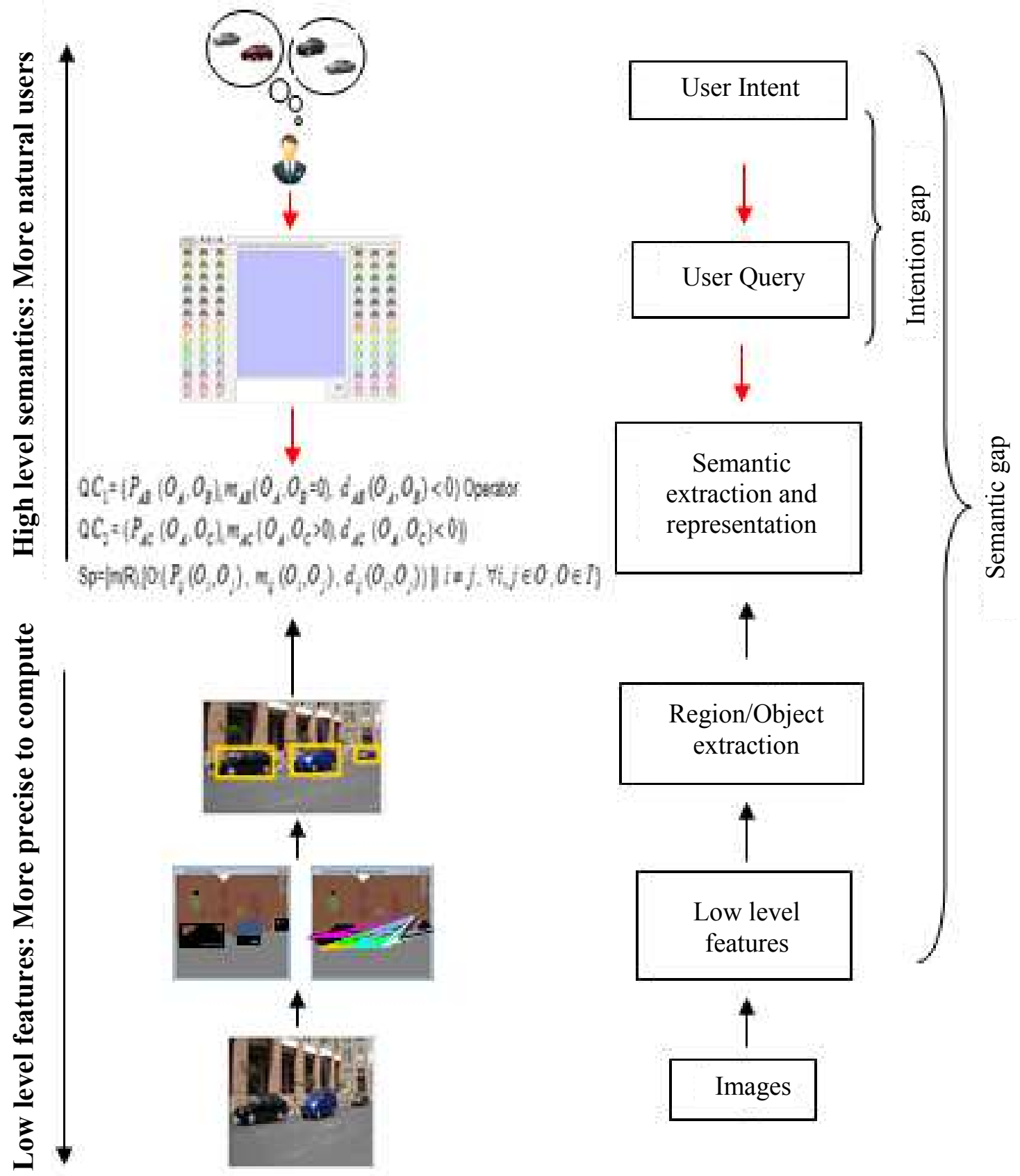

Fig. 1: Semantic and intention gaps-semantic extraction and representation of traffic images 
Friendly user interface should be straightforward, easy to use and satisfying user's various searching needs (Zhang, 2010). The ability to retrieve images based on image semantic content is a clear priority (Eakins et al., 2004) for image database user while embracing the rich image descriptions as opposed to the limited information returned by using short search queries. The fundamental idea of image semantics should be reflected in the searches besides employing image attributes in user search query, whereas user can compare the relevance of image results based on the semantic features that differentiate between the retrieved images (Wang et al., 2015; Westman, 2009).

A novel image search method named as Semantic Visual Query Builder (SeVQer) is implemented, which not only addresses the intention gap but also reduces the semantic gap in general. Different to conventional image search methods which uses an image as query, image search in SeVQer allow user to formulate his/her query image by selecting multiple objects with various visual attributes and semantic spatial relationships. The performance of the SevQer is evaluated for image search and retrieval and the experimental results demonstrate its robustness and effectiveness.

This paper begins with the related work, follows by description on the architecture and components in SeVQer. Next, the data set used, experiment setup and metrics for performance evaluation are explained before discussing the results and analyses. The paper is concluded in the final section.

\section{Related Work}

An effective user interface will allows the users to accurately express their search need and intent when searching for images that fitted their perception (Hu et al., 1999) should be integrated on top of any image retrieval system. Image search has been intensively studied (Flickner et al., 1995; Lew et al., 2006; Niblack et al., 1993). Two main approaches in finding images based on user intent from image databases are browsing and searching.

For image browsing approach, the users browse through the images from the entire collection. Most of the browsing models (Pečenovió et al., 2000; Chen et al., 2000a; Chen et al., 1998; Descampe et al., 2007; Bederson, 2001; Chen et al., 2000b) arrange the images into a hierarchy or structure that can be browsed interactively by users. The greatest challenge of a browsing approach is to identify structures that contribute to effective search. These structures are beneficial in supporting fast navigation, browsing path selection and reduce the browsing domain to only an area of interest (Cox, 1992). Image browsing is inefficient and time consuming when users are not familiar with the structure of the image collection unknown. Thus, image searching using query by visual example has been introduced.

A famous paradigm commonly used to describe the perceptual aspects of the visual features of image content is query by visual example (Cox et al., 2000; Kelly et al., 1995; Tieu and Viola, 2000). The visual queries are based on the formulation of low level image features extracted during image extraction stage. Correspondingly, finding a suitable visual example as search query may be a difficult task itself (Rodden, 1999). To tackle the issue, efforts have been made to extend the search query either by selecting a local region as search query (Cheng et al., 1998; Carson et al., 1997) or by sketching the search query (Kato et al., 1992; Daoudi and Matusiak, 2000; Chan et al., 1997; Lai, 2000; Egenhofer, 1997). For the formal, users can select the region of interest in the query image by placing a boundary box or manually trace the boundary (depends on functionality offer by the search system). The latter is known as query by sketch, which lets users to draw or sketch the desired mental picture with image editor software. However, drawing what's in the human mind is also difficult for the retriever and an image drawing which must be recognized by the system, is a major restriction for retrieval. Another major difficulty is when users want to retrieve an ambiguous target image especially for describing an abstract and object spatial relationship images. User usually prefers to use keywords to indicate their search intent (Kherfi et al., 2004; Fu, 2007) but using keywords as image search query only works well when images in the database are annotated with accurate and standardized textual information. On the contrary, there are times and situations when users are unable to express what they had in mind into precise and correct wording (Zha et al., 2010; Niblack et al., 1993; Gerrig and Zimbardo, 2001). Besides, short keyword-based queries are usually ambiguous and exclusion of critical keywords would thus unable to fully reflect users' intents precisely (Zha et al., 2010).

Regardless of image browsing or searching approaches, both no clear target image and no alternative human interface provided contributed inefficiency in finding user intent matching search results. Hence, understanding a user's query intent is important for achieving higher search accuracy and thus improving user satisfaction. The representation of spatial relations semantics among objects are important as it can convey important information about the image and to further increase the confidence in image understanding which will contribute to richer querying and retrieval facilities (Wang, 2013). Besides, semantic representation that can accurately represent the intent of the input query to satisfy a user's information need and thus for image retrieval based semantic concepts are also needed (Hu et al., 2009). 


\section{Architecture of SeVQer}

In this section, the details of SeVQer are described. Firstly, an overview of the architecture is given and followed by the introduction on the flows and details of the user query design.

\section{Overview}

In performing effective image retrieval, a user already knows what (s) he is looking for, but it may be difficult to articulate their intention into words. In this study, the SeVQer architecture allows the learning of the user's preferences to guide in determining what the user is looking for through the interaction and browsing session. The five main modules are shown in Fig. 2 where each component is described briefly.

The first module is the Visual Query Builder where users are allowed in the formulation of intent specific queries by dragging and dropping their interested objects with the visual attribute as well as their semantic spatial relationships among the objects. The second module is the Semantic Visual Query Builder which automatically extracts semantic meaning of the user query in the user query builder. Third module consist of the Semantic
Database Builder which automatically extracts and identifies the semantic meaning of images which capture the object and spatial relationship among objects in the images. Fourth module is the Semantic Image Similarity use to define spatial similarity between the image in the database and the user query. The fifth module is the Image Database that stores all the extracted semantic features.

\section{User Query}

Both design of Semantic Color Perception and User Query Builder are contributing to building an intuitive and visual user query design that allows a user to express their need easily for the image retrieval. Processes involved in the user query design are described below.

\section{Semantic Color Name}

The number of semantic color is based on the HSV color setting. There are eleven semantic color chosen as color representation in the vehicle colors. They are red, orange, yellow, blue, pink, white, grey, green, brown, purple and black. The semantic color names are customizable by users. User can change the semantic color name by selecting and clicking a color where the potential color name will be listed in a panel as in Fig. 3.

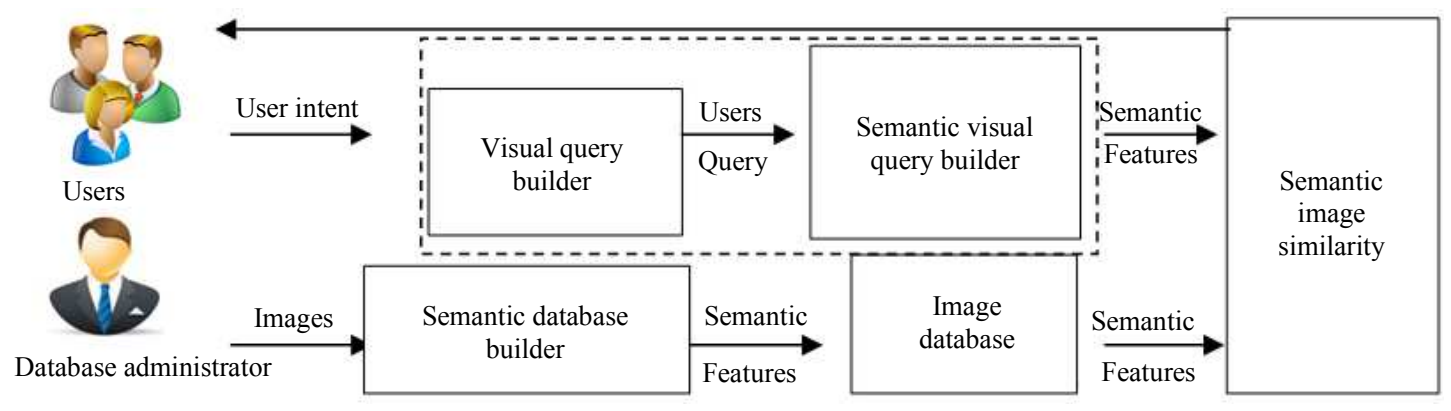

Fig. 2: The SeVQer architecture

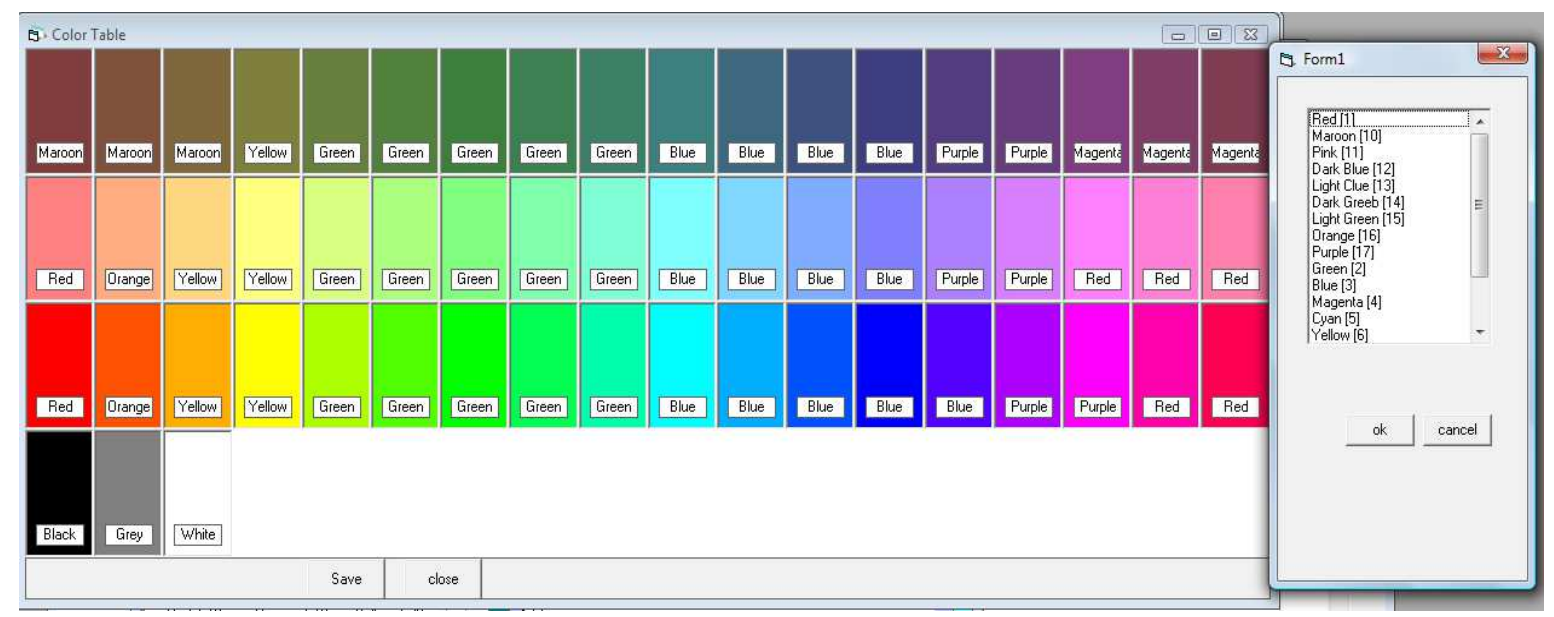

Fig. 3: Semantic color name 


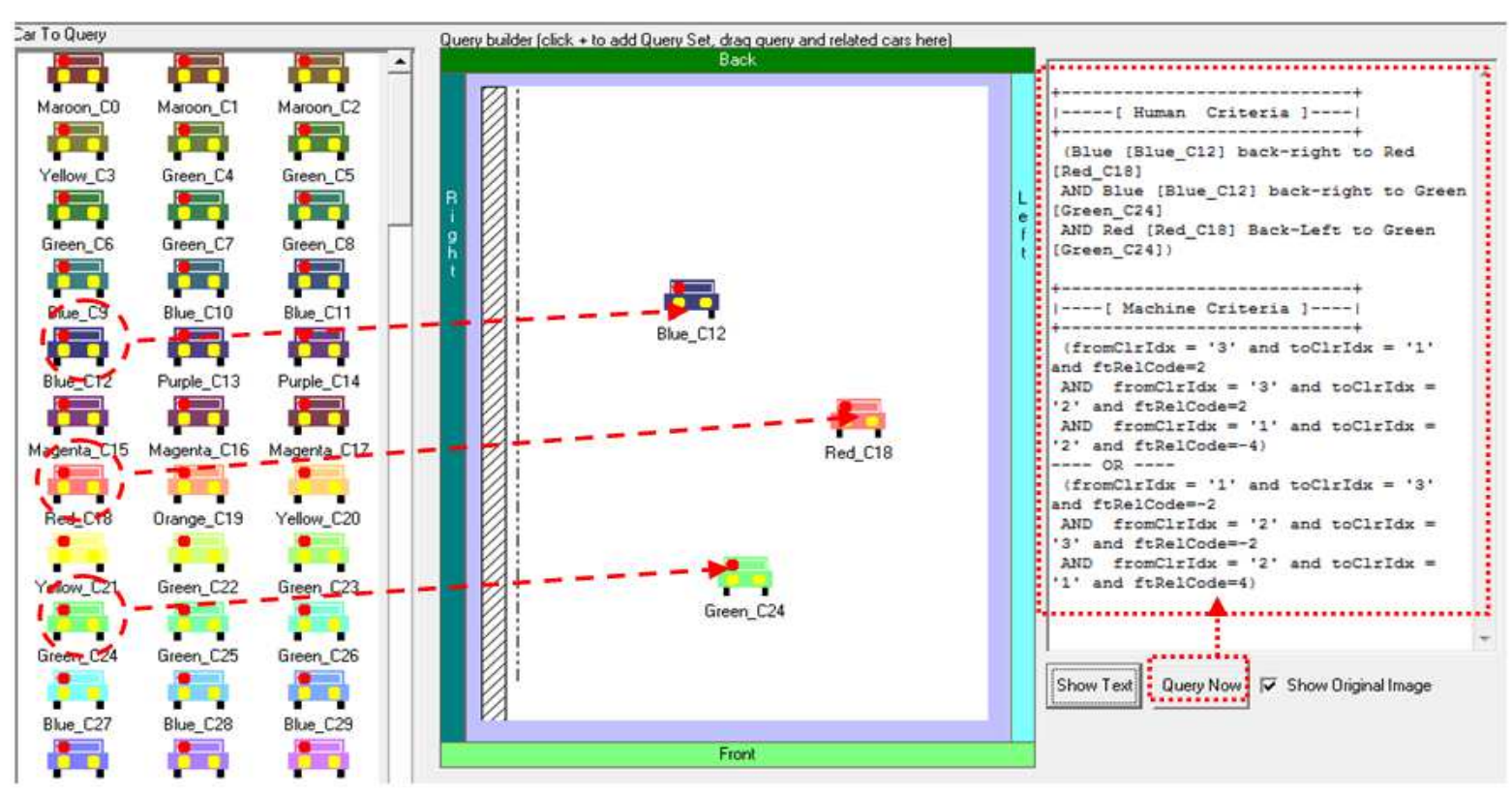

Fig. 4: The visual user query handler

\section{Visual Query Handler}

For image retrieval, the user needs to formulate intent specific queries. The screenshot of a sample query using the visual user query builder is shown in Fig. 4. SeVQer image search interface allows the drag and drop of objects from the left panel to formulate user query in the middle panel. Objects with the interested visual attribute (i.e., color) as well as the semantic spatial relationship (i.e., front or back, left or right) among the objects formed the user query. Clicking on the "Show Text" button generates the semantic features in the right panel while the retrieval process is initiated by clicking the "Query Now" button.

\section{Image Browsing}

Screen-shot of image browsing is shown in Fig. 5. User can browse all images in the database by selecting any image from the list box in the left panel. User selected image is shown in the right panel. Objects in selected image are extracted and represented in human perception view in the top-middle panel. The main objects are represented as cars and their spatial relationship are represented as per position shown. The semantic features of these objects are indicated in the middle-bottom panel.

\section{Experiment and Evaluation}

An image search interface named the Semantic Visual Query Builder (SeVQer) is implemented to demonstrate the feasibility of a non-verbal interface.
For experiments, the efficiency of the SeVQer is compared with three image search methods (searchby-example, search-by-sketch and search-bykeyword). Then, the acceptability and subjective satisfaction of the implemented image search interface of SeVQer by a group of participants is conducted. It is also ensured that the intended users of a system can carry out the intended tasks efficiently and satisfactorily. All experiments are performed on a Window XP operating system, $2.0 \mathrm{GHz}$ processor with 1 GB memory. All the components are implemented with Microsoft Visual Basic 6.0.

\section{Data Set}

A total of 130 low complex scene of traffic images with perspective view are created and used for the empirical evaluation. All images are in the sizes of $512 \times 512$ pixels. The vehicles in the traffic images have forever changing spatial relationships that can be captured to contain a variety of vehicles (objects of interest) with their spatial relationships in the images. In this data set, all the private information is removed and each object (vehicle) is identified by its visual attributes.

\section{Procedure}

Sample images are provided and displayed on the SeVQer image search interface to avoid the difficulty of interpreting from an image to verbal keywords in keyword-based image retrieval or producing a correct drawing of an image in sketch-based image retrieval. The users can formulate intent specific queries by dragging and dropping objects with the interested 
visual attribute together with the semantic spatial relationship among the objects of interest visualized on the screen. This functionality assists in expressing user's search intents more precisely, mimicking the mental image in the user's brain. The semantic features are automatically generated after user express their user query in the visual user query builder. Only images that contain the query objects that satisfy both spatial and logical constraints are answered. All interactions with the image search interface are conducted with the use of a computer mouse.

\section{Experiment Setup}

The performance of four different image search methods are experimented and measured (search-bykeyword, search-by-example, search-by-sketch and the implemented image search-by-SeVQer). A total of 60 participants from a local university, aged 21-28 participated in the experiments. Majority of participants never have any experience with any kind of CBIR systems. 15 participants are randomly assigned to test on all four tasks illustrating the scenarios using one of the image search methods. Refer to Table 1 for the four traffic image scenarios and task description.

For task 1 and 2, the targets are two cars without and with spatial relationship. Participants are instructed to illustrate Scenario 1 and 2 based on the sample provided in the visual user query builder for searching two cars of the same and different color, without and with their spatial relationship. For task 3 and 4, the targets are multiple cars with their spatial relationship involved. Participants are requested to illustrate Scenario 3 and 4 based on the sample provided in visual user query builder for searching multiple cars of different color and same color with spatial relationship.

Table 1: The four scenario and average task completion time

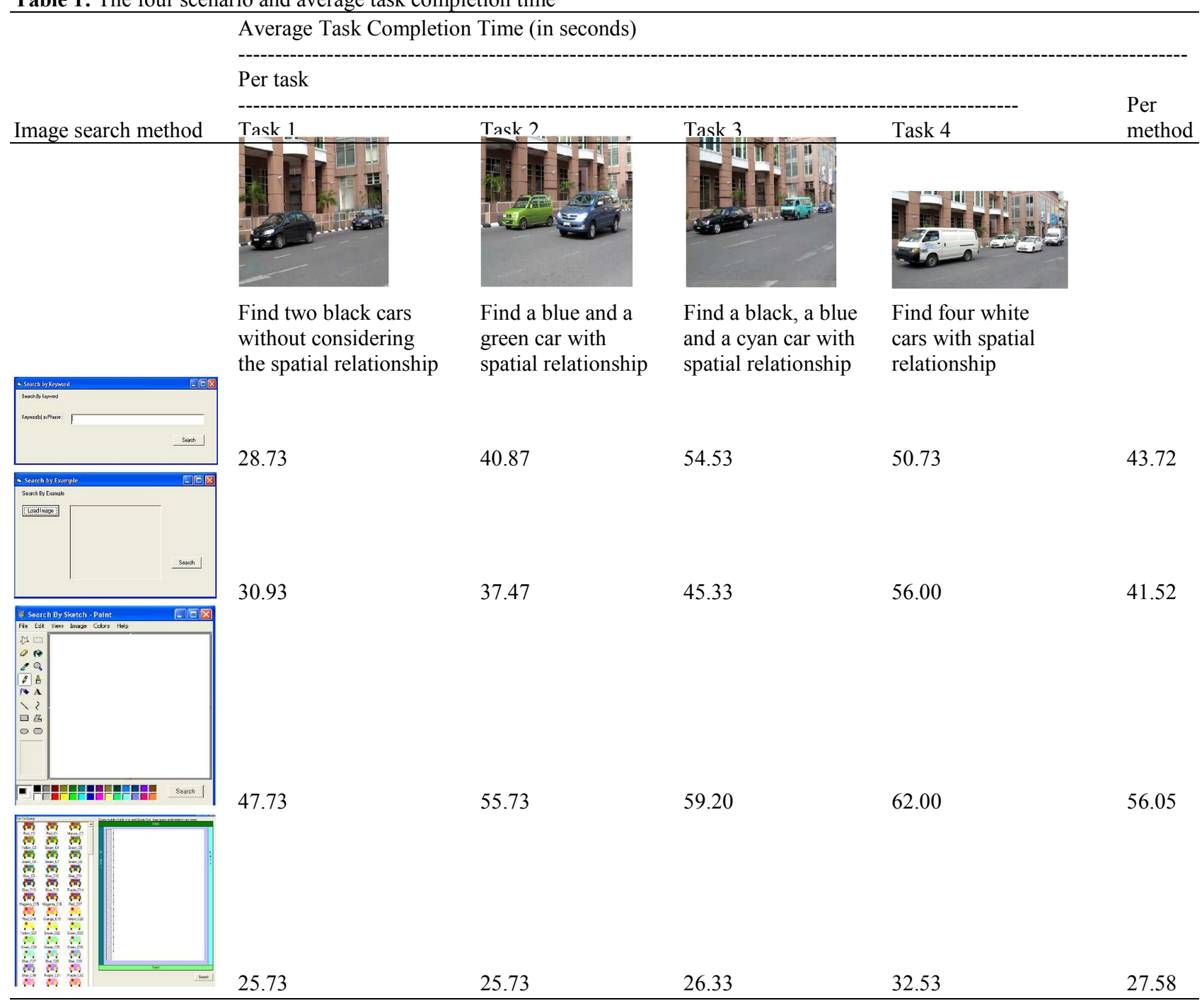




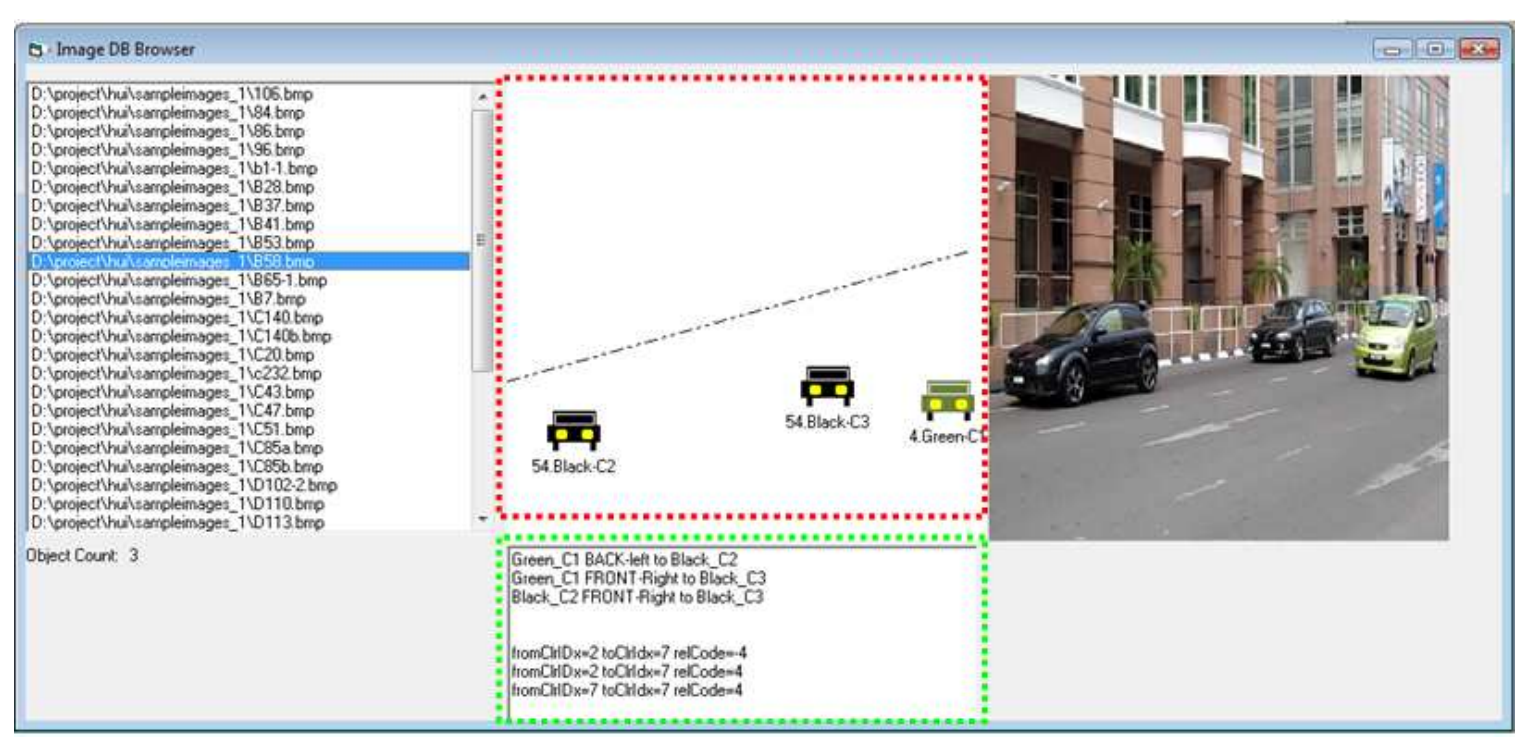

Fig. 5: Image browsing

Participants are firstly trained with sample scenario before actual experimentation. Participants are required to complete all tasks using the randomly assigned image search method as accurate as possible with the shortest time. Participants are not allowed to ask question during the sessions.

\section{Metrics}

Two measurements used to evaluate SeVQer are efficiency (task completion time) and user satisfaction. Efficiency is measured based on the time taken (in seconds) to complete the given task. If a participant completes a task faster using method $\mathrm{X}$ than method $\mathrm{Y}$, then method $\mathrm{X}$ is more efficient than method $\mathrm{Y}$. User satisfaction level is measured based on a 9-point score. The participants are asked to rate their satisfaction based on the provided image search methods. The rate ranged from 1: "strongly dissatisfied" to 9: "strongly satisfied" based on the aspect of user interface friendliness to find traffic image (Question A) and the agreement to use again the image search methods for future image searching (Question B). For testing statistical significance, single factor ANOVA in the software of Statistical Package for the Social Sciences (SPSS, 2008) is used. The independent variables for the experiment are the four image search methods. The dependent variables are task completion time recorded.

\section{Results and Analyses}

In this section, performance of the implemented SeVQer method with three existing image search methods (search-by-example, search-by-sketch and search-by-keyword) was compared and discussed. All the participants performed four different tasks based on the assigned image search method. The task completion time of the participants are recorded. All participants answered a post-questionnaire with a 9point score on satisfaction level after completing all the tasks. All participants can complete all search tasks successfully. Collected data from the experiment was analyzed using SPSS.

\section{Efficiency}

A single factor ANOVA was conducted to compare the effect of different traffic image search methods for all four tasks. There was a significant effect of image search methods on task completion time at the $\mathrm{p}<0.05$ level for the four tasks $[F(3,56)=67.98, p=0.00]$. A Tukey post hoc test revealed that the task completion time for the traffic image search was statistically significantly lower using SeVQer $(27.58 \pm 4.29 \mathrm{sec}, \mathrm{p}=$ $0.00)$ while image search-by-sketch required highest task completion time $(56.17 \pm 4.83, \mathrm{p}=0.00)$. There was no statistically significant difference between the search-by-example and search-by-keyword $(p=0.919)$. Taken together, these results suggest that traffic image search by SeVQer do affect task completion time.

As reported in Table 1, an average of $56.05 \mathrm{sec}$ is required to complete the given task using sketch query, followed by an average of 43.72 sec using keyword query. Participants had difficulty and confusion in verbalizing the scenario in words especially in terms of describing the color and spatial relationship of the objects. Image search-by-example has an average of $41.52 \mathrm{sec}$, which is only $2.20 \mathrm{sec}$ less than the average time with keyword(s) query. However, participants need more time when the collection of images increased or when they cannot express the user query where no intent image is provided in the database. 
Table 2: The average subjective satisfaction

\begin{tabular}{lll} 
& QA & QB \\
Image search method & $\begin{array}{l}\text { Do you think it was easy and user friendly } \\
\text { to find photo using this image search? }\end{array}$ & $\begin{array}{l}\text { I would choose this image search } \\
\text { method for image searching in future. }\end{array}$ \\
\hline Keyword & 6.07 & 6.47 \\
Example & 6.53 & 6.47 \\
Sketch & 6.00 & 6.20 \\
SeVQer & 7.73 & 7.73 \\
\hline
\end{tabular}

Comparatively, search by the implemented SeVQer are more efficient where participants spent the shortest time in completing the task with an average time of $27.58 \mathrm{sec}$. Therefore, participants performed more efficiently using SeQVer image search interface as compared to other methods. The average task time per task is shown in Table 1. It is noticed that the task completion time for task 3 and 4 are much longer than task 1 and 2 because task 3 and 4 are more complicated as their involved more objects and pairs of spatial relationships.

\section{User Satisfaction}

A single factor ANOVA was conducted to compare the subjective satisfaction of different traffic image search methods. There was a significant effect of image search methods on user satisfaction at the $\mathrm{p}<0.05$ level for QA $[\mathrm{F}(3,56)=8.78, \mathrm{p}=0.000]$ and $\mathrm{QB}[\mathrm{F}(3,56)=$ $6.57, p=0.001]$. For both questions, post hoc Tukey test revealed that user satisfaction between SeVQer and the other 3 methods differed significantly at $p<0.05$ while there was no significant relationship between the 3 image search methods.

Participants felt more satisfied with SeVQer (mean satisfaction of 7.73) for both questions as compared to other image search methods (refer to Table 2). The mean user satisfaction for querying image using search-byexample is 6.53 for QA followed by 6.07 for querying image using search-by-keyword on the same question. Both query using search-by-example and search-bykeyword have the same mean satisfaction of 6.47 on QB. The least satisfied method is the image search query search-by-sketch where the mean satisfaction is 6.00 and 6.20 for QA and QB respectively.

Taken together, the results suggested that SeVQer performs better than other image search methods (search-by-keyword, search-by-example and search-bysketch) tested in terms of efficiency and user satisfaction. On the ability to complete the given search tasks, SeVQer is more efficient than other query search methods because it allows participants to illustrate the search scenario faster, hence, less time spent to complete the search tasks. Keywords search is time consuming as participants faced difficulty and confusion in describing the scenario in words especially describing color and spatial relationship of the objects. Efficiency for query search by-example decreases when the image database is of high volume and when images in the database are unable to represent the intended user query. Query search by-sketch is least efficient among all the search methods as more time to express and represent the user query where knowledge in basic drawing using computer is also required. Therefore, SeVQer is more efficient compared to other query search methods. Also, participants rated SeVQer with higher user satisfaction rate compared to other query search methods in term of easy to use and user friendliness.

\section{Conclusion}

The implemented image search of Semantic Visual Query Builder (SeVQer) was designed for users to easily express their specific search need with matching intent that is capable to interpret and extract the semantic features of user query automatically. The usability and efficiency of the system are confirmed by the experiments conducted. The results showed that the implemented semantic visual user query achieved shortest completion time and highest in user satisfaction. The implemented semantic visual user query builder can be a powerful image search interface for CBIR, especially when the user has no clear a priori target image or has difficulty expressing the target image verbally. By these design, user can formulate intent specific queries by dragging and dropping their interested objects with the visual attribute as well as their semantic spatial relationships among the objects instead of the heavy mental load of visualizing an ambiguous image.

\section{Acknowledgement}

This research was funded by Universiti Malaysia Sarawak under F08/SpFRGS/1528/2017

\section{Author's Contributions}

All authors equally contributed in this work.

\section{Ethics}

The article is original and contains unpublished material. The corresponding author confirms that all the other authors have read the manuscript and approved it for publication. 


\section{References}

Bederson, B.B., 2001. PhotoMesa: A Zoomable Image Browser using Quantum Treemaps and Bubblemaps. Proceedings of the 14th Annual ACM Symposium on User Interface Software and Technology, Nov. 11-14, Orlando, FL, USA, pp: 71-80. DOI: $10.1145 / 502348.502359$

Belkin, N., 2000. Helping people find what they don't know. Commun. ACM, 43: 58-61. DOI: $10.1145 / 345124.345143$

Belkin, N., D. Kelly, G. Kim, J.Y. Kim and H.J. Lee et al., 2003. Query length in interactive information retrieval. Proceedings of the 26th Annual International ACM SIGIR Conference on Research and Development in Information Retrieval, Jul. 28-Aug. 1, Toronto, Canada, pp: 205-212.

DOI: $10.1145 / 860435.860474$

Carson, C., S. Belongie, H. Greenspan and J. Malik, 1997. Region-based image querying. Proceedings of the IEEE Workshop on Content-Based Access of Image and Video Libraries, Jun. 20-20, IEEE Xplore Press, St Thomas, U.S. Virgin Islands, USA, pp: 42-49. DOI: 10.1109/IVL.1997.629719

Chan, Y., Z. Lei, D. Lopresti and S. Kung, 1997. A feature-based approach for image retrieval by sketch. Proceedings of the SPIE 3229, Multimedia Storage and Archiving Systems II, (SAS' 97), SPIE, pp: 220-231. DOI: 10.1117/12.290343

Chang, C.Y., H.Y. Wang and C.F. Li, 2009. Semantic analysis of real world images using support vector machine. Expert Syst. Applic., 36: 10560-10569. DOI: 10.1016/j.eswa.2009.03.041

Chen, C., G. Gagaudakis and P. Rosin, 2000a. Similarity-based image browsing. Proceedings of the 16th IFIP World Computer Congress (International Conference on Intelligent Information Processing), Aug. 22-22, Beijing, China, pp: 206-213.

Chen, J.Y., C.A. Bouman and J.C. Dalton, $2000 \mathrm{~b}$. Hierarchical browsing and search of large image databases. IEEE Trans. Image Process., 9: 442-455. DOI: $10.1109 / 83.826781$

Chen, J.Y., C.A. Bouman and J.C. Dalton, 1998. Similarity pyramids for browsing and organization of large image databases. Proceedings of the SPIE 3299, Human Vision and Electronic Imaging III, (VEI' 98), SPIE, pp: 563-575.

DOI: $10.1117 / 12.320147$

Cheng, S.F., W. Chen and H. Sundaram, 1998. Semantic visual templates: Linking visual features to semantics. Proceedings of the International Conference on Image Processing, Oct. 7-7, IEEE Xplore Press, Chicago, IL, USA, pp: 531-535. DOI: 10.1109/ICIP.1998.727321
Choi, Y. and E.M. Rasmussen, 2003. Searching for images: The analysis of users' queries for image retrieval in American history. J. Am. Society Inform. Sci. Technol., 54: 498-511. DOI: 10.1002 asi.10237

Choi, Y., 2010. Effects of contextual factors on image searching on the Web. J. Am. Society Inform. Sci. Technol., 61: 2011-2028. DOI: 10.1002/asi.21386

Cox, I., M. Miller, T. Minka, T. Papathomas and P. Yianilos, 2000. The Bayesian image retrieval system, PicHunter: theory, implementation and psychophysical experiments. IEEE Trans. Image Process., 9: 1057-7149. DOI: 10.1109/83.817596

Cox, K., 1992. Information retrieval by browsing. Proceedings of the 5th International Conference on New Information Technology, (NIT' 92), Hong Kong, pp: 69-80.

Croft, W.B. and R.H. Thompson, 1987. $I^{3} R$ : A new approach to the design of document retrieval systems. J. Am. Society Inform. Sci., 38: 389-404. DOI: $10.1002 /($ SICI) $1097-$ 4571(198711)38:6<389::AID-ASI1>3.0.CO;2-4

Daoudi, M. and S. Matusiak, 2000. Visual image retrieval by multiscale description of user sketches. J. Visual Lang. Comput., 11: 287-301. DOI: $10.1006 /$ jvlc. 2000.0159

Descampe, A., C. De Vleeschouwer, M. Iregui, B. Macq and F. Marqués, 2007. Prefetching and caching strategies for remote and interactive browsing of JPEG2000 images. IEEE Trans. Image Process., 16: 1339-1354. DOI: 10.1109/TIP.2007.894258

Eakins, J., P. Briggs and B. Burford, 2004. Image Retrieval Interfaces: A User Perspective. In: Image and Video Retrieval, Enser, P., Y. Kompatsiaris, N.E. O’Connor, A.F. Smeaton and A.W.M. Smeulders (Eds.), Springer Berlin Heidelberg, pp: 628-637.

Egenhofer, M., 1997. Query processing in spatial-queryby-sketch. J. Visual Lang. Comput., 8: 403-424. DOI: 10.1006/jvlc.1997.0054

Flickner, M., H. Saywhney, W. Niblack, J. Ashley and Q. Huang et al., 1995. Query by image and video content: The QBIC system. Computer, 28: 23-32. DOI: $10.1109 / 2.410146$

$\mathrm{Fu}, \mathrm{H} ., 2007$. Attention-driven image interpretation, annotation and retrieval. Unpublished dissertation in partial fulfillment of the requirements for the degree of Doctor or Philosophy, Hong Kong Polytechnic University, Hong Kong.

Gerrig, R. and P. Zimbardo, 2001. Psychology and Life. 16 Edn., Allyn and Bacon, ISBN-10: 020533511X, pp: 704.

Gudivada, V.N. and V.V. Raghavan, 1995. Contentbased image retrieval systems. IEEE Comput., 28: 18-22. DOI: $10.1109 / 2.410145$ 
Hu, J., G. Wang, F. Lochovsky, J.T. Sun and Z. Chen, 2009. Understanding user's query intent with Wikipedia. Proceedings of the 18th International Conference on World Wide Web, Apr. 20-24, Madrid, Spain, pp: 471-480.

DOI: $10.1145 / 1526709.1526773$

Hu, P.J.H., P.C. Ma and P. Chau, 1999. Evaluation of user interface designs for information retrieval systems: A computer-based experiment. Decision Support Syst., 27: 125-143.

DOI: $10.1016 / \mathrm{S} 0167-9236(99) 00040-8$

Jansen, B., A. Spink and T. Saracevic, 2000. Real life, real users and real needs: A study and analysis of user queries on the web. Inform. Process. Manage., 36: 207-227. DOI: 10.1016/S0306-4573(99)00056-4

Kato, T., T. Kurita, N. Otsu and K. Hirata, 1992. A sketch retrieval method for full color image database-query by visual example. Proceedings of the 11th International Conference on Patter Recognition. Conference A: Computer Vision and Applications, Aug. 30-Sept. 3, IEEE Xplore Press, The Hague, Holland, pp: 530-533. DOI: $10.1109 /$ ICPR.1992.201616

Kelly, D. and X. Fu, 2007. Eliciting better information need descriptions from users of information search systems. Inform. Process. Manage., 43: 30-46. DOI: 10.1016/j.ipm.2006.03.006

Kelly, P., T. Cannon and D. Hush, 1995. Query by image example: The Comparison algorithm for Navigating Digital Image Databases (CANDID) approach. Proceedings of the SPIE 2420, Storage and Retrieval for Image and Video Databases III, (IVD' 95), SPIE, pp: 238-248. DOI: $10.1117 / 12.2052089$

Kherfi, M., D. Ziou and A. Bernardi, 2004. Image retrieval from the world wide web: Issues, techniques and systems. ACM Comput. Surveys, 36: 35-67. DOI: 10.1145/1013208.1013210

Lai, T., 2000. CHROMA: A photographic image retrieval system. Unpublished dissertation in partial fulfillment of the requirements for the degree of Doctor or Philosophy, University of Sunderland, Sunderland, United Kingdom.

Lew, M., N. Sebe, C. Djeraba and R. Jain, 2006. Content-based multimedia information retrieval: State of the art and challenges. ACM Trans. Multimedia Comput. Commun. Applic., 2: 1-19. DOI: $10.1145 / 1126004.1126005$

Ménard, E., 2011. Search behaviours of image users: A pilot study on museum objects. Partnership: Canad. J. Library Inform. Pract. Res., 6: 1-18. DOI: 10.21083/partnership.v6i1.1433
Nakazato, M., L. Manola and T. Huang, 2003. Image Grouper: Search, Annotate and Organize Images by Groups. In: Recent Advances in Visual Information Systems, Chang, S., Z. Chen and S. Lee (Eds.), Springer Berlin Heidelberg, pp: 129-142.

Niblack, W., R. Barber, W. Equitz, M. Flickner and E. Glasman et al., 1993. The QBIC project: Querying images by content using color, texture and shape. Proceedings of the SPIE 1908, Storage and Retrieval for Image and Video Databases, (IVD' 93), SPIE, pp: 173-187. DOI: 10.1117/12.143648

Pečenovió, Z., M.N. Do, M. Vetterli and P. Pu, 2000. Integrated Browsing and Searching of Large Image Collections. In: Advances in Visual Information Systems, Laurini, R. (Ed.), Springer Berlin Heidelberg, pp: 279-289.

Rodden, K., 1999. How do people organise their photographs? Proceedings of the 21st Annual BCSIRSG Conference on Information Retrieval Research, Apr. 19-20, eWiC, Glasgow, pp: 12-12.

Santini, S. and R. Jain, 2000. Integrated browsing and querying for image databases. IEEE Multimedia, 7: 26-39. DOI: 10.1109/93.879766

Smeulders, W., M. Worring, S. Santini, A. Gupta and R. Jain, 2000. Content-based image retrieval at the end of the early years. IEEE Trans. Patt. Anal. Machine Intell., 22: 1349-1380. DOI: 10.1109/34.895972

SPSS, 2008. SPSS statistics for windows, Version 17.0. SPSS Inc., Chicago.

Tieu, K. and P. Viola, 2000. Boosting image retrieval. Proceedings of the IEEE Conference on Computer Vision and Pattern Recognition, Jun. 15-15, IEEE Xplore Press, Hilton Head Island, SC, USA, pp: 228-235. DOI: 10.1109/CVPR.2000.855824

Urban, J. and J. Jose, 2006. An Explorative Study of Interface Support for Image Searching. Proceedings of the 3rd International Workshop on Adaptive Multimedia Retrieval, Jul. 28-29, Springer, Glasgow, UK, pp: 207-221. DOI: $10.1007 / 1167083417$

Wang, H.H., 2013. Performance and analysis of the automated semantic object and spatial relationships extraction in traffic images. Adv. Comput. Sci.: Int. J., 2: 37-42.

Wang, H.H., D. Mohamad and N.A. Ismail, 2015. Towards semantic user query: A review. J. Comput. Sci., 11: 1017-1024. DOI: $10.3844 /$ jcssp.2015.1017.1024

Wang, Z., D. Feng, Z. Chi and T. Xia, 2006. Annotating image regions using spatial context. Proceedings of 8th IEEE International Symposium on Multimedia, Dec. 11-13, IEEE Xplore Press, San Diego, CA, pp: 55-61. DOI: 10.1109/ISM.2006.32 
Westman, S., 2009. Image Users' Needs and Searching Behaviour. In: Information Retrieval: Searching in the 21st Century, Göker A. and J. Davies (Eds.), John Wiley and Sons, Ltd. Chichester, UK.

Yoon, J., 2011. Searching images in daily life. Library Inform. Sci. Res., 33: 269-275.

DOI: 10.1016/j.lisr.2011.02.003

Zha, Z., L. Yang, T. Mei, M. Wang and Z. Wang et al., 2010. Visual query suggestion: Towards capturing user intent in internet image search. ACM Trans. Multimedia Comput. Commun. Applic., 6: 1-19. DOI: $10.1145 / 1823746.1823747$
Zhang, D., 2010. A survey of user interfaces in contentbased image search engines on the web. Proceedings of the 4th Workshop on Human Computer Interaction and Information Retrieval, Aug. 22-22, New Brunswick, New Jersey, pp: 129-132. 